\title{
Modified Naiver-Stokes equation for conceptual tests of pure field physics
}

\author{
Igor E. Bulyzhenkov ${ }^{1,2, a}$ \\ ${ }^{1}$ Moscow Institute of Physics and Technology, 9 Institutskiy per., Dolgoprudny, Moscow reg., 141700, Russia \\ ${ }^{2}$ Lebedev Physics Institute RAS, 53 Lininsky pros., Moscow, 119991, Russia
}

\begin{abstract}
Cartesian relativistic physics has its own nondual analog of the 1915 Einstein Equation for pure field physics in nonempty space. This tensor field analog leads to the vector geodesic equations for relativistic accelerations of Ricci material densities. Extended states of inertial energy densities modify the Navier-Stokes equation by the kinematic 'living forces' for the slow motion of material media. The new inertial feedback enables a conceptual choice between Newtonian and Cartesian alternatives (with localized or extended, respectively, elementary masses in the Universe) because of different pressure and temperature gradients across laboratory flows of liquids and gases.
\end{abstract}

\section{Introduction}

Cartesian physics $[1,2]$ of material space is currently described only in qualitative terms and without quantitative predictions of specific phenomena for the theory verification/falsification procedure. The concept of void space regions without matter was unclear to Descartes, who after Aristotle also maintained the extension of matter or 'matter-extension' as the continuous material plenum. The Cartesian vortex mechanics for all types of observable spatial displacements in this material plenum was published in 1629. Later Newton's successful dynamics of point-like masses shook the need in sophisticated matter-extensions with vortex states. Nowadays, Newton's mechanics of localized masses and continuous gravitation fields in empty space forms the dual core of contemporary space theories in the Solar system and beyond. Not Cartesian physics, but Newtonian empty space modeling became mandatory for 1916 Einstein's gravitation in the weak field limit. No one looked at mechanical theories if they did not fit Newton's mass transport at low speeds.

Dual classical physics for spatially separated matter and fields is traditionally assigned to the macroscopic world, while physicists tend to assign nondual material densities (or quantum fields) only to the microscopic world. But the unique physical reality is either dual or nondual regardless of the spatial scaling and mathematical formalisms in available theories. Similarly, the matter is either local or non-local regardless of suitable approaches to describe it. The celebrated Einstein-PodolskyRosen paradox initiated the long-term discussion and many tests of the material world nonlocality. Now nonlocality of matter is reasonably considered beyond quantum physics [3]. The world holism [4] and the global direct overlap of all material elements motivates our attempts to develop nonNewtonian mechanics for mass densities of the extended particle. There is no in reality a mesoscopic

\footnotetext{
ae-mail: bulyzhenkov.ie@mipt.ru
} 
scale for possible transitions from nondual microscopic physics to the dual macroscopic model of Newton. Material states should initially be considered in nondual field terms on micro-, macro-, and mega-scales in qualitative, if not quantitative, approaches to any energy flows in the Universe.

The purpose of this paper is to trace the balanced energy origin of extended sources in Einstein's metric gravitation, to study the strong field compensation for positive kinetic and negative potential self-energies of equal inertial and gravitational masses, to propose a nondual field analogue of the Einstein Equation, and to predict new laboratory phenomena for conceptual tests of extended matter physics. Based on propositions of Aristotle, Descartes, Mie [5] and Einstein [6], we shall try to study how to redesign classical relativistic gravitation in pure field terms - heavy material fields without localized particles. Our initial idea is that 1938 Einstein-Infeld physics may coherently replace the supposed empty space (between the localized charges) with the Aristotle space plenum of moving material fields. This may assist to give up the unnecessary concept of Newton's point-like particles and to modify the old building of classical gravitation by strong field solutions without energy divergence and metric singularities in the center of gravitational sources.

The Einstein Equation in the dual (field + matter) Newtonian paradigm has been known since 1915. The similar tensor equation in the Cartesian paradigm of the united space-matter operates with a nondual field continuum of extended mass-energies. This continuum is filled by overlapping radial vortexes with chaotic auto-rotations of elementary metric densities. Such permanent metric motions within the elementary Cartesian vortex reveal the kinematic origin of the rest-mass energy $m c^{2}$. This active, kinetic energy may be called the internal relativistic heat. The positive (kinetic, active) energy is always accompanied by equal, but negative (potential, passive) self-energy according to the principle of energy self-compensation (introduced below). There is no elementary inertial mass without vortex auto-rotations of metric space. And circular material densities with positive kinetic energy generate self-gravitation with negative (passive) elementary energies of Cartesian vortex states that results in the global compensation of active and passive energies in the non-empty space Universe.

We will return to the Einstein idea that the geodesic equations of motion should follow from the strong-filed gravitation equation. This was difficult to prove from iterations in the dual post-Newtonian physics. But Cartesian geodesic relations for Ricci material densities can be derived exactly from the nondual field analogue of the Einstein Equation. In order to demonstrate some practical benefits of non-empty space physics, we will modify the Navier-Stokes equation in terms of Ricci energy flows with the inertial feedback of 'living forces', $-\mu \partial_{i} V^{2} / 2$. This dynamical pressure averts asymptotically divergent energy flows and can initiate conceptual tests of Newton vs Cartesian mechanics in the laboratory.

\section{Einstein's material fields for 1629 Cartesian vortex mechanics}

The predominant majority of people believe that electric charges and inertial masses are located within visible frames of macroscopic bodies. Such bodies can be divided into smaller parts in accordance with everyday observations. Thus, the smallest part of substance was introduced as a so-called corpuscle (the point particle in the indivisible limit) that is a carrier of the elementary mass and charge. Classical fields between localized particles are considered massless and chargeless. Nevertheless, the experiment is only a criterion of truth, but not the truth itself. The materialistic pragmatism of highly educated and well-equipped researchers of the supposed dual 'reality' (particle and field are different notions) was rejected by many philosophers. Indeed, by dividing an infinitely extended field-energy object into parts one should again obtain only infinite objects rather than corpuscles of limited size.

In contrast to available observations, nonempty space of continuous material flows has been recognized by philosophers not only in the Ancient East and in Ancient Greece, but also by many contemporary thinkers in the West (Mie, Einstein, Infeld) and in Russia (Fedorov, Umov, Vernadsky, Roerich 
et al.[7]). "A coherent field theory requires that all elements be continuous... And from this requirement arises the fact that the material particle has no place as a basic concept in a field theory. Thus, even apart from the fact that it does not include gravitation, Maxwell's theory cannot be considered as a complete theory"'as was stated by Einstein and Infeld [6] in 1938. Indeed, the postulated pointparticle paradigm results in Coulomb energy divergence, which terminates Classical Electrodynamics as a self-consistent theory. A point source in the Maxwell-Lorentz Equations may be considered as "an attempt which we have called intellectually unsatisfying" according to De Broglie [8]. Einstein also criticized his 1915 field equation for the point gravitational source: "it resembles a building with one wing built of resplendent marble and the other built of cheap wood" (translation [9]). The Dirac delta operator for the density of point matter in the void seems to have pushed physicists away from physical reality and the Eastern approaches to superimposed energy flows.

The evolution of Einstein's theory of relativity has already passed three milestones. They are the 1905 postulates and the internal energy $m c^{2}$ of mechanical bodies, the 1915 geometrization of massless metric fields in the Einstein Equation under the Newton's empty space dogma, and the 1938 proposition to distribute particle's mass-energy $m c^{2}$ continuously over all spatial points of the material metric field in the non-Newton nondual approach to physical reality. Recall that the integration of particles into spatial structures of their fields was suggested by Einstein together with Infeld [6] for the further evolution of all natural disciplines: "We would regard matter as being made up of regions of space in which the field is extremely intense... There would be no room in this new physics for both field and matter, for the field would be the only reality." However, the extended mass has not been yet adopted by modern relativists. Their Newtonian references traditionally associate General Relativity (GR) only with the 1916 empty space metric of Schwarzschild, later denied by Einstein for physical reality in the 1939 thought experiment [10]. And they use the Dirac delta-function for the formal presentation of the Lagrange material density $\mu(\mathbf{x})=m \delta(\mathbf{x}-\boldsymbol{\xi}) / \sqrt{\gamma}$ in the point mass action $S=-c \int m d s(\boldsymbol{\xi})=-c \iiint \int \mu(\mathbf{x}) d s(\mathbf{x}) \sqrt{\gamma} d^{3} x=-c \iiint \int \mu(\mathbf{x})\left(d s / \sqrt{g}_{o o} d x^{o}\right) \sqrt{\gamma} \sqrt{g}_{o o} d^{4} x$ for the Newton empty space $x^{i}$ with material peculiarities along the path $\boldsymbol{\xi}\left(x^{o}\right)$.

The elementary mass density is to be a continuous spatial function in the Cartesian world and in the Einstein-Infeld pure field approach to matter [6]. According to Einstein, there are equal inertial (active $\mu_{a}$ ) and gravitational (passive $\mu_{p}$ ) masses. Today the best mathematical candidate in nonempty pseudo-Riemannian world to match the sum of active and passive mass-energy densities is the Ricci scalar $R \equiv g^{\mu \nu} R_{\mu \nu}=\left(\mu_{a}+\mu_{p}\right) c^{2} / \zeta \varphi_{o}^{2}[11,12]$. Here $\varphi_{o}=c^{2} / \sqrt{G}=1.04 \times 10^{27} V$ is the universal potential for the inertial/gravitational mass-energy charge $q_{m} \equiv E_{m} / \varphi_{o}=\sqrt{G} m$ and $\zeta=1 / 8 \pi$. The symmetrical Ricci tensor $R_{\mu \nu}=\partial_{\lambda} \Gamma_{\mu \nu}^{\lambda}-\Gamma_{\mu \rho}^{\lambda} \Gamma_{v \lambda}^{\rho}+\Gamma_{\mu \nu}^{\lambda} \partial_{\lambda} \ln \sqrt{g_{o o}}-\partial_{\mu} \partial_{\nu} \ln \sqrt{g_{o o}}$ was already found for the static metric of nonempty space, when $\sqrt{-g}=\sqrt{g_{o o}}, g_{o i}=0$, and $\partial_{o} g_{o o}=0$. Indeed, the distributed static mass-energy $M c^{2}$ warps $g_{o o}(r)=1 / g^{o o}(r)=\left[1-U_{o}(r) E_{p}^{-1}\right]^{-2}=\left(1+r_{o} / r\right)^{-2}$ for a probe mass-energy $E_{p}$ at its potential energy $U_{o}(r)=-G M E_{p} / r c^{2}=-r_{o} E_{p} / r$. Only two warped connections $\Gamma_{o o}^{i}=\partial_{i} g_{o o} / 2$ and $\Gamma_{i o}^{o}=\partial_{i} g_{o o} / 2 g_{o o}$, when $\partial_{o} g_{\mu v}=0$ and $U_{i}=0$, define $R_{o}^{o}=g^{o o} R_{o o}=$ $g^{o o}\left(\partial_{i} \Gamma_{o o}^{i}-\Gamma_{o o}^{j} \Gamma_{o j}^{o}\right)=\left[-\partial_{i}^{2} \ln \left(g_{o o}^{-1 / 2}\right)+\left(\partial_{i} \ln \left(g_{o o}^{-1 / 2}\right)\right)^{2}\right]$ and $R=g^{o o} R_{o o}+g^{i j} R_{i j}=g^{o o}\left(\partial_{i} \Gamma_{o o}^{i}-\Gamma_{o o}^{j} \Gamma_{o j}^{o}\right)-$ $\delta^{i j}\left(-\partial_{j} \Gamma_{o i}^{o}-\Gamma_{i o}^{o} \Gamma_{j o}^{o}\right)=2 R_{o}^{o}$. This geometrical formalism for static gravitational fields reveals the Ricci scalar meaning as a sum of passive and active mass densities,

$$
\frac{\varphi_{o}^{2} R(r)}{8 \pi}=\frac{\varphi_{o}^{2} R_{o}^{o}(r)}{4 \pi}=\frac{\vec{w}^{2}(r)}{4 \pi}+\frac{\left(-\varphi_{o}\right) \nabla \vec{w}(r)}{4 \pi} \equiv \mu_{a}(r) c^{2}+\mu_{p}(r) c^{2},
$$

where $\vec{w}(r)=-\nabla W(r), W=-\varphi_{o} \ln \left(1+r_{o} / r\right), \mu_{a}=\mu_{p}=m r_{o} / 4 \pi r^{2}\left(r+r_{o}\right)^{2}$, and $G_{o}^{o}=R_{o}^{o}-R / 2=0$.

Cartesian vortex dynamics can be developed on the basis of vortex mechanical fields $\varphi_{o} f_{\mu \nu} \equiv$ $\varphi_{o}\left(\nabla_{\mu} u_{v}-\nabla_{\nu} u_{\mu}\right)$ from the local four-velocity $u_{\mu}=g_{\mu \nu} d x^{\nu} / d s$ of moving material space. Local mechanical and gravitational mass-energies originate from local vortex motion of this space, with the 
Principle of Equivalence for active and passive masses and with the mutual compensation of active (kinetic, positive) and passive (gravitational, negative) self-energies under such a mechanical motion. The relativistic invariant for the active (positive) mass-energy density of vortex mechanical space is $\left(-\varphi_{o}^{2} f_{\mu v} f^{\mu v} / 8 \pi\right.$ ), which is together with its passive (yin-yang) partner is distributed continuously over all elementary 4-volumes $\sqrt{-g} d^{4} x \equiv \sqrt{g_{o o}} d x^{o} \sqrt{\gamma} d^{3} x$ in the action integral for the 'void nothing':

$$
\begin{array}{r}
S=-\frac{\varphi_{o}^{2}}{16 \pi c} \iiint \int\left(-f_{\mu v} f^{\mu v}+f_{\mu v} f^{\mu v}\right) d s \sqrt{\gamma} d^{3} x \equiv \\
-\frac{\varphi_{o}^{2}}{16 \pi c} \iiint \int\left(-f_{\mu \nu} f^{\mu v}+\frac{2 u_{v} \nabla_{\mu}\left(f^{\nu \mu} B\right)}{B}\right) B \sqrt{-g} d^{4} x-\frac{\varphi_{o}^{2}}{8 \pi c} \iiint \int \partial_{\mu}\left(\sqrt{-g} B u_{v} f^{\mu v}\right) d^{4} x .
\end{array}
$$

Here we introduced the kinematic scalar field $B(x)=d s(x) / \sqrt{g_{o o}} d x^{o}$ next to the active and passive mass densities in the space-time elementary volume $\sqrt{-g} d^{4} x$,

$$
B(x) \equiv \frac{\sqrt{g_{\mu \nu} d x^{\mu} d x^{v}}}{\sqrt{g_{o o}} d x^{o}} \equiv \frac{1}{\sqrt{g_{o o}} u^{o}} \equiv \frac{\sqrt{1-\beta^{2}}}{1-g_{o i} v^{i} / \sqrt{g_{o o}}} \equiv \sqrt{1-\beta^{2}}\left(1+\frac{g_{o i} d x^{i}}{g_{o o} d x^{o}}\right) .
$$

This inertial field depends on the GR metric tensor $g_{\mu \nu}=g_{v \mu}$, the physical three-velocity $v^{i} \equiv$ $c d x^{i} / \sqrt{g_{o o}} d x^{o}\left[1+\left(g_{o i} d x^{i} / g_{o o} d x^{o}\right)\right]$ and the relativistic speed factor $\beta^{2} \equiv \gamma_{i j} v^{i} v^{j} / c^{2}$, with $\gamma_{\mu \nu} \equiv$ $g_{o \mu} g_{o v} g_{o o}^{-1}-g_{\mu v}$. The inertial field $B$ contributes to the four-current of mass-energy,

$$
J^{\mu}(x) \equiv 4 \pi \mu(x) c^{2} u^{\mu} B(x) \equiv 4 \pi \mu(x) c^{2} \frac{d x^{\mu}}{\sqrt{g_{o o}} d x^{o}}=\varphi_{o}^{2} \frac{\partial_{v}\left(\sqrt{-g} B f^{\mu v}\right)}{\sqrt{-g}} .
$$

This Maxwell-type equation is the equality due to the Einstein equality of active and passive mass densities. The variational equation (4) supports the complex charge unification [13] of extended massenergies with imaginary electric self-energies, where $E=(\sqrt{G} m+i e) \varphi_{o}=511 \mathrm{KeV}-i 104 \times 10^{22} \mathrm{KeV}$ for the electron.

By applying the Einstein Principle of Equivalence to moving densities of active and passive masses in (2), one can say from (4) that the mass density $\mu(x)$ of extended matter in Cartesian mechanics originates from vortex velocities of material space:

$$
\mu(x)=-\frac{\varphi_{o}^{2}}{8 \pi c^{2}}\left(\partial_{\mu} u_{v}-\partial_{\nu} u_{\mu}\right)\left(\nabla^{\mu} u^{v}-\nabla^{v} u^{\mu}\right) .
$$

Relations (4)-(5) for the rest frame of the extended elementary particle without net rotations lead $\mu(r)=m r_{o} / 4 \pi r^{2}\left(r+r_{o}\right)^{2}$ due to the following metric components $g_{o o}=r^{2} /\left(r+r_{o}\right)^{2}, r_{o} \equiv \sqrt{G} m / \varphi_{o}=$ $G m / c^{2}, \gamma_{i j}=\delta_{i j}$, and $g^{i j}=-\delta^{i j}[11]$.

Despite inertial (kinetic) and gravitational (potential) mass densities are equal in (4), the positive kinetic $\left(+\mu_{a} c^{2}\right)$ and the negative potential self-energies have opposite signs. The gravitational self-action always arises due to the intense interaction potential [11-13] $W(r) \equiv \varphi_{o} \ln \sqrt{-g}=$ $\varphi_{o} \ln \sqrt{g_{o o}(r)}=-\varphi_{o} \ln \left[\left(r+r_{o}\right) / r\right]$ of the emerged charge density $\sqrt{G} \mu_{p}$, with $\mu_{p} \equiv \mu_{a}$ :

$$
E_{k i n}+U_{s e l f}=\int\left[\mu_{a}(r) c^{2}-\sqrt{G} \mu_{g r}(r) \varphi_{o} \ln \frac{1}{\sqrt{g_{o o}(r)}}\right] \sqrt{\gamma} d^{3} x=\left(m_{a}-m_{p}\right) c^{2} \equiv 0 .
$$

Here the energy density is negative only in the very core of the radial carrier and is positive for $r>r_{o} /(e-1)$. The integral energy balance (6) means the exact mutual compensation of kinetic and potential self-energies of any rest-mass body due to the Einstein Principle of Equivalence for 
inertial $\left(m_{i n}\right)$ and heavy $\left(m_{g r}\right)$ masses. Contrary to measurable exchanges of internal and translation kinetic energies, the negative potential energy $\left(-m_{g r} c^{2}\right)$ is not relevant to observations in practice. But negative energy tensions within the continuous carrier (of the elementary relativistic heat $Q=m c^{2}$ ) is relevant to stability of the elementary extended mass-energy by the internal negative pressure, once assumed by Poincare for the extended particle.

\section{Nondual Einstein-type equation for moving material space}

The internal heat, as well as an internal kinetic degree of freedom under the spatial transport of elementary energy, cannot be reasonably assigned to the Newton point mass. Therefore, the variable internal heat is the principle difference between Newton and Einstein transport of energy, even at the low speed motion of mechanical bodies. Indeed, the Newton point particle possesses only one degree for the summary (internal and translation) kinetic energy, while the Cartesian distributed vortex for the elementary heat-energy possesses both kinetic internal and kinetic translation energies. The Special Relativity kinematic cooling $Q\left(\beta^{2}\right)=Q_{o} \sqrt{1-\beta^{2}} \approx\left(m c^{2}-m v^{2} / 2-m v^{4} / 8 c^{2}-\ldots\right)$ of the elementary rest-mass energy $m c^{2} \equiv Q_{o}$, which is the elementary relativistic heat, and the pure translation (mechanical) energy $p_{i} v^{i} \equiv H\left(\beta^{2}\right)+L\left(\beta^{2}\right)=Q_{o} / \sqrt{1-\beta^{2}}-Q_{o} \sqrt{1-\beta^{2}} \approx\left(m v^{2}+m v^{4} / 2 c^{2}+\ldots\right)$ reitirate together the Newton-type summary changes of internal (heat) and external (mechanical, translation) kinetic energies, $\Delta\left[Q\left(\beta^{2}\right)+p_{i} v^{i}\right]=\Delta\left(m v^{2} / 2+3 m v^{4} / 8 c^{2}+\ldots\right)$ for the low speed $\left(\beta^{2} \ll 1\right)$ transport of the elementary energy carrier. There is no mass transpart and conservation in relativistic energy physics.

The slow spatial motion of the Cartesian mechanical body is first of all the transport of the variable heat integral $Q_{o}\left(1-\beta^{2} / 2\right)$ of vortex densities. The accompanying fraction of the kinetic energy $Q_{o} \beta^{2}$ due to the translation ordering is very small compared to the kinetic energy of internal chaos. Cartesian transport of two variable energies just formally correspond to the Newton transport of the constant mass $Q_{o} / c^{2}$ plus one variable energy $Q_{o} \beta^{2} / 2$. Newton had no idea about the internal degree of kinematic heat-energy and modeled transport of net energy changes, $\left[Q_{o}\left(1-\beta^{2} / 2\right)+Q_{o} \beta^{2}\right]-Q_{o}=$ $Q_{o} \beta^{2} / 2$, through one summary coefficient $Q_{o} / 2 c^{2} \equiv m / 2$. It is clear from Cartesian physics that Newton's model works properly only for small probe bodies in empty space where internal (heat) and external (translation) kinematic degrees of freedom obey the collinear transport.

In condensed media, where heat energy gradients and mechanical energy flows may have different directions, Cartesian dynamics with internal degrees of freedom may not reiterate Newtonian one. Moreover, the Newton model of motionless point masses without internal heat does not coincide with the Cartesian system of static vortices which initially have internal kinetic energy or heat. One can expect some principle differences between the dual and nondual theories of matter for its spatial acceleration and its heat transfer in liquids and gases. Here, different exchange mechanisms can control non-Newtonian energy gains, $m v^{2}$, and thermal losses, $-m v^{2} / 2$, of the relativistic kinematic cooling. Early or later, the elementary relativistic energy of internal vortex motions with variable heat should replace the Newtonian constant mass as a basic notion for description of warm material space in General Relativity. The latter can not rely on Newton's cold masses in order to incorporate thermodynamics at the low speed limit. Equally, metric gravitation of cold masses cannot be considered as a true limit for gravitation of Cartesian energy-charges, which depend on variable heat.

The last integral in the 'void nothing' action (2) can be replaced by integration of 4-forces $u_{v} f^{\mu v} B$ over the hyper-surface that provide the internal Poincare pressure for stabilization of the extended mass. The other part of the action integral is related to active and passive mass densities, which we 
describe through the Ricci scalar R,

$$
\frac{\varphi_{o}^{2} R(x)}{16 \pi c^{2}}=\mu(x)=\frac{\mu_{a}(x)+\mu_{p}(x)}{2}=-\frac{\varphi_{o}^{2} f_{\mu v} f^{\mu v}}{16 \pi c^{2}}+\frac{\mu u_{\mu} u^{\mu}}{2} .
$$

Now we perform the Hilbert metric variations over $\delta g^{\mu \nu}$ in (2), $\delta S=$ $-\left(\varphi_{o}^{2} / 16 \pi c\right) \int d^{4} x \delta(\sqrt{-} g B R)-\left(\varphi_{o}^{2} / 8 \pi c\right) \int d^{4} x \partial_{\mu} \delta\left(\sqrt{-g} B u_{v} f^{\mu v}\right) \equiv \int \sqrt{-g} d^{4} x T_{\mu \nu} \delta g^{\mu \nu} / 2 c=0$, in order to derive a nondual analog of the Einstein Equation for pure field physics of moving material flows,

$$
T_{\mu \nu} \equiv \zeta \varphi_{o}^{2}\left[\left(u_{\mu} u_{v}-\gamma_{\mu \nu}\right) \frac{B R}{2}-B R_{\mu \nu}+\nabla_{\mu} \nabla_{\nu} B-g_{\mu \nu} \nabla_{\lambda} \nabla^{\lambda} B\right]+P_{\mu \nu} \equiv 0 .
$$

The Ricci material flows of active and passive mass densities are balanced in (8) by the Poincare negative stress $P_{\mu \nu}$, which originates from the Hilbert variations of local force densities $\delta\left(\sqrt{-g} B u_{v} f^{\mu \nu}\right) / \delta^{\mu \nu}$.

Einstein was the first who tried to derive the motion of particles directly from his gravitational field equation. He was engaged into this conceptual problem for more than 20 years. Empty space regions in Newtonian dual physics are described by field solutions with zero Ricci curvatures, $R_{\mu v}=0$. The motion of point peculiarities in such massless fields can be approximated only through successive iterations [14-16]. Cartesian physics of material fields is free from these complicated iteration procedures. One can find the exact geodesic equations for any non-equilibrium motion of inertial Ricci densities in the world metric space-time as the vector flow conservation from the general tensor balance (8), $\nabla_{v} T_{\mu}^{v}=0$. Here it is sufficient to make use of the known geometrical equalities $\nabla_{\nu} \nabla_{\mu} \nabla^{v} B-\nabla_{\mu} \nabla_{v} \nabla^{v} B \equiv R_{\mu \nu} \nabla^{v} B \equiv R_{\mu}^{v} \nabla_{v} B$ and the Bianch identities $2 \nabla_{v} R_{\mu}^{v} \equiv \nabla_{\mu} R$ :

$$
\frac{\varphi_{o}^{2}}{16 \pi}\left[\nabla_{\nu}\left(B R u_{\mu} u^{\nu}\right)-B \nabla_{\mu} R-\gamma_{\mu \lambda} \nabla^{\lambda}(B R)\right]=-\nabla_{\nu} P_{\mu}^{\nu} .
$$

This general equation of motion can be equally read for the 4-acceleration $u^{\nu} \nabla_{\nu} u^{\mu}$ along a normal axis to the 4-velocity $u^{\mu}$ of the scalar mass density $\mu=\varphi_{o}^{2} R / 16 \pi c^{2}$,

$$
B \mu c^{2} u^{\nu} \nabla_{v} u^{\mu}+\left(u^{\mu} u^{v}-g^{\mu v}\right)\left[\frac{g_{o v}}{g_{o o}} \nabla_{o}(\mu B)-\mu \nabla_{v} B\right] c^{2}=\left(u^{\mu} u^{v}-g^{\mu v}\right) \nabla_{\lambda} P_{v}^{\lambda} .
$$

The vector equation (10) for Cartesian mechanics claims that the inertial field $B(x) \neq$ const contributes to geodesic dynamics of material space densities, while dual theories with the Newton empty space alternative are free from such kinematic feedbacks.

\section{Modified Navier-Stokes equation for Cartesian physics}

It is very difficult to infer from observations and measurements whether the world space is empty, as Newton modeled for localized bodies and point particles, or this space is filled continuously everywhere with invisible matter, as Aristotle and Descartes claimed. The experimental procedure of a theory verification can reject improper approximations of reality, but cannot select the most correct approach among suitable alternatives [17]. For instance, there are about ten competing formulations of Quantum Mechanics at the moment. General Relativity of 1916 was requested to reiterate Newton's empty space dogma. But Einstein himself began to share the Cartesian matter-extension for the pure field physics at least since 1938. And non-empty space metric constructions for Einstein's General Relativity lead again to the same post-Newton findings for the main gravitational tests [12] as the empty-space metric of Schwarzschild can propose. What is the use of reinforcing the AristotleDescartes material space if it might not be ever justified in practice? 
Recall again that Newton's low speed dynamics works satisfactorily for small probe bodies where transports of the internal kinetic energy or relativistic heat $Q=m c^{2}\left(1-\beta^{2} / 2\right)$ and the kinetic energy of spatial translations $m c^{2} \beta^{2}$ have collinear directions. Newtonian proponents have to consider the mechanical motion as a transport of the constant elementary mass $m=$ const plus the transport of one kinetic energy $m v^{2} / 2$. They derive dynamical laws for acceleration of such constant masses and, basing on these laws, compute accompanying energy flows. Cartesian physicists have to consider first of all the transport of elementary vortex energies with the kinematic cooling under motion, $m c^{2}\left(1-\beta^{2} / 2\right) \neq$ const, and they have to derive dynamical laws for vector energy flows. There is no mass transport and mass conservation law in cartesian physics. Basing on dynamical laws for variable heat and translation energies, one can also model the accompanying transport of elementary objects with the rest-frame energy $m c^{2}$. The competing force and energy approaches lead to the same low speed dynamics of small probe energies $m c^{2}$ in external fields but not of material densities $\mu$ within continuous media with inhomogeneous 'life forces' $\mu c^{2} \beta^{2} / 2$. Streams of liquids and gases with heat gradients do not obey Newton's dynamic for cold masses and appropriate conceptual tests can distinguish Newton and Cartesian world organizations in the regular Earth laboratory.

The Cartesian acceleration law (10) for mass-energy flows within the Einstein-Infeld material space can be considered in the limit of vanishing gravitational fields, $g_{\mu \nu} \rightarrow \eta_{\mu \nu} \equiv\{1,-1,-1,-1\}$, and low speeds, $\beta^{2} \rightarrow 0, B \rightarrow 1-\beta^{2} / 2, v^{i} \rightarrow c d x^{i} / d x^{o} \equiv V^{i}=V_{i}, d V^{i} / d t=\partial_{t} V^{i}+V^{j} \partial_{j} V^{i}=$ $\partial_{t} V^{i}+\delta^{i j} \partial_{j} V^{2} / 2-[\mathbf{V} \times \operatorname{curl} \mathbf{V}]^{i}$ :

$$
\mu\left(\partial_{t} V^{i}+V^{j} \partial_{j} V^{i}\right)+V^{i} \partial_{t} \mu+\frac{\mu \partial_{i} V^{2}}{2}=-\partial_{i} p+\mu v \partial_{j} \partial^{j} V^{i}+f^{i}
$$

Here the post Euler force density $V^{i} \partial_{t} \mu$ is the 1903 Tsiolkovsky force for the reactive rocket motion. The new kinematic feedback $\mu \partial_{i} V^{2} / 2$ originates from local gradients of the scalar inertial field (3), which is not relevant to the 1916 GR dynamics in Newtonian empty space. The new kinematic potential $V^{2} / 2$, which modifies Euler's fluid dynamics and the Navier - Stokes equation, appears in (11) exclusively in Cartesian physics due to the nondual tensor equation (8) for the nonempty inertial space. The right-hand side of the equation (11) is associated with net forces from the stress tensor $P_{\mu}^{v}$ in (10). Here we underlined the Euler's equation force with the pressure $p$ gradient and the simplest drag force with the Stokes kinematic viscosity $v$ next to all other possible forces $f^{i}$.

The pressure $p=\mu\left(s T+\mu_{c h}-\varepsilon\right)$ within fluids can be bound quantitatively, as is known [18, 19], with the temperature $T$, the specific entropy $s$, the chemical potential $\mu_{c h}$, and the specific internal energy $\varepsilon$, with $d \varepsilon=T d s-p d(1 / \mu)$ and $d \mu_{c h}=(d p / \mu)-s d T$. The pressure differential, $d p=(s T+$ $\left.\mu_{c h}-\varepsilon\right) d \mu+\mu\left(s d T+d \mu_{c h}+p d \mu^{-1}\right)=\mu\left(s d T+d \mu_{c h}\right)$, is related only to changes of the local temperature and the chemical potential. The net dynamical balance, $d V_{i} / d t=-s \partial_{i} T-\partial_{i}\left(\mu_{c h}+V^{2} / 2\right)-V_{i} \partial_{t} \ln \mu+$ $v \partial_{j} \partial^{j} V_{i}$, of the Euler material derivative $d V_{i} / d t$ in the equation (11) depends on speed gradients next to thermal ones. Such a modification can suggest a lot of conceptual tests to compare Newtonian and Cartesian physics even for steady flows of liquids and gases with $d V_{i} / d t=0$. For example, contrary to Newtonian physics for laminar fluids with homogeneous pressure and temperature across the radial section of tubes, Cartesian physics predicts inhomogeneous $p(r)$ and $T(r)$ across the same steady flows. This can shed new light on 'unexpected' heat and pressure on boundaries of many dynamical systems like rocket engines, Ranque-Hilsch vortex tubes [20], plasma beams, etc. There are also various applications of the modified Navier-Stokes equation (11) to turbulent flows of energy in industrial hydro generators of electric power systems.

The kinematic self-deceleration $-\partial_{i} V^{2} / 2$ due to the feedback of Leibniz - de Coriolis 'living forces' of a moving material space can be understood only through the Cartesian approach to physical reality. This inertial self-deceleration prevents asymptotic flows with diverging en- 
ergy densities. The unphysical energy divergence in the original Euler and Navier-Stokes equations was questioned by many researchers, including experts of the Clay Mathematical Institute (http://www.claymath.org/millennium-problems/navier-stokes-equation). Newtonian based mechanics results in the incomplete description of Navier-Stokes flows, while the Cartesian replica (11) with the inertial damping by 'living forces' is a more realistic approximation of real fluid dynamics.

In general, the new inertial force with its kinematic potential (3) for moving material spaces can justify the nondual field reality of Einstein and Infeld for the macroscopic Cartesian world. Nondual extended matter refutes applications of Newtonian dynamics to continuous media and considers the Navier - Stokes equation with diverging asymptotic solutions as conceptually insolvent. By closing, Cartesian mechanics does deserve very careful investigation for low speed energy flows and for further technological developments in a line of the Einstein and Infeld physics of the pure field.

\section{References}

[1] D. Garber, Descartes's Metaphysical Physics (University of Chicago Press, Chicago, 1992)

[2] Descartes's Physics. In The Cambridge Companion to Descartes, eds J. Cottingham (Cambridge University Press, New York, 1992) 286-334

[3] S. Popescu, Nonlocality beyond quantum mechanics. Nature Physics 10, 264-270 (2014)

[4] J.C. Smuts, Holism and Evolution. 2nd Edition (Macmillian and Co, London, 1927)

[5] G. Mie, Grundlagen einer Theorie der Materie. Ann. der Physik.37, 511-534 (1912); ibid 39, 1-40 (1912); 40, 1-65 (1913)

[6] A. Einstein, L. Infeld, The Evolution of Physics (Cambridge Press, Cambridge, 1938)

[7] G.M. Young, The Russian Cosmists: The Esoteric Futurism of Nikolai Fedorov and His Followers(Oxford UP, New York, 2012)

[8] L. de Broglie, New perspectives in physics (Basic Books, New York, 1962)

[9] M.-A. Tonnelat, The principles of electromagnetic theory and relativity (Riedel Publishing Co., Dordrecht, 1966)

[10] A. Einstein, On a Stationary System With Spherical Symmetry Consisting of Many Gravitating Masses. Annals of Mathematics 40, 922-936 (1939)

[11] I.E. Bulyzhenkov, Einstein's Gravitation for Machian Relativism of Nonlocal Energy-Charges. Int. Jour. of Theor. Phys. 47, 1261-1269 (2008)

[12] I.E. Bulyzhenkov, Geometrization of Radial Particles in Non-Empty Space Complies with Tests of General Relativity. Jour. Mod. Phys. 3, 1465-1478 (2012)

[13] I.E. Bulyzhenkov, Complex charge densities unify particles with fields and gravitation with electricity. Bulletin Lebedev Phys. Inst, 43, 138-142 (2016)

[14] A. Einstein, L. Infeld, \& B. Hoffmann, The Gravitational Equations and the Problem of Motion. Ann. Math.39, 65-100 (1939)

[15] A. Einstein, L. Infeld, The Gravitational Equations and the Problem of Motion. II. Ann. Math. 41, 455-564 (1940)

[16] A. Einstein, L. Infeld, On the motion of particles in general relativity theory. Canad. Jour. Math. 1, 209-241 (1949)

[17] K. Popper, The Logic of Scientific Discovery (Hutchinson \& Co,London and New York, 1959)

[18] S.J. Putterman, Superfluid Hydrodynamics (Elsevier, New York, 1974)

[19] L.D. Landau, E.M. Lifshitz, Fluid Mechanics (Elsevier, 2013)

[20] S. Eiamsa-arda, P. Promvongeb, Review of Ranque-Hilsch effects in vortex tubes. Renewable and Sustainable Energy Reviews 12, 1822-1842 (2008) 